\title{
Nabothian Cyst
}

National Cancer Institute

\section{Source}

National Cancer Institute. Nabothian Cyst. NCI Thesaurus. Code C34835.

A benign, mucus-filled cervical cyst that occurs when mucus-secreting columnar epithelial cells are covered with squamous epithelium. 